\title{
TWEAK Promotes 0steoclastogenesis in Rheumatoid Arthritis
}

\author{
Jin-Sil Park, ${ }^{*}$ Seung-Ki Kwok, ${ }^{*}$ Mi-Ae Lim, ${ }^{*}$ Hye-Joa Oh, ${ }^{*}$ Eun-Kyung Kim, ${ }^{*}$ Joo-Yeon Jhun, ${ }^{*}$ Ji Hyeon Ju, ${ }^{*}$ Kyung-Su Park, \\ Young-Woo Park, ${ }^{\dagger}$ Sung-Hwan Park, ${ }^{*}$ Ho-Youn Kim, ${ }^{*}$ Young-Gyu Cho, ${ }^{\ddagger}$ and Mi-La Cho*
}

\begin{abstract}
From The Rheumatism Research Center, * Catholic Research Institute of Medical Science, The Catholic University of Korea, Seoul; the Integrative Omics Research Center, ${ }^{\dagger}$ Korea Research Institute of Bioscience and Biotechnology, Daejeon; and the R\&D Center/Research Group $3,{ }^{\ddagger}$ Handok Pharmaceuticals Co, Ltd, Seoul, South Korea
\end{abstract}

\author{
Accepted for publication \\ May 10, 2013. \\ Address correspondence to \\ Mi-La Cho, Ph.D., Rheumatism \\ Research Center, Catholic \\ Institutes of Medical Science, \\ The Catholic University of \\ Korea, 222 Banpo-Daero, \\ Seocho-gu, Seoul 137-701, \\ South Korea; or Young-Gyu \\ Cho, Ph.D., R\&D Center/ \\ Research Group 3, Handok \\ Pharmaceuticals Co, Ltd, Seoul, \\ South Korea. E-mail: \\ iammila@catholic.ac.kr or \\ younggyu.cho@handok.com.
}

\begin{abstract}
Bone destruction is critical in the functional disability of patients with rheumatoid arthritis (RA). Osteoclasts, specialized bone-resorbing cells regulated by cytokines, such as receptor activator of $N F-\kappa B$ ligand (RANKL), are primarily implicated in bone destruction in RA. The aim of the study was to examine whether tumor necrosis factor-like weak inducer of apoptosis (TWEAK), a member of the tumor necrosis factor superfamily, has osteoclastogenic activity in patients with RA and in animal models, including mice with collagen-induced arthritis (CIA) and IL-1 receptor antagonist knockout (IL-1RaK0) mice. TWEAK was increased in the synovium, synovial fluid, and serum of patients with RA and in the synovium of CIA mice and IL-1RaKO mice. TWEAK induced RANKL expression in mixed joint cells and splenocytes from CIA mice, IL-1RaKO mice, and fibroblast-like synoviocytes from patients with RA. Both osteoclast precursor cells and osteoclasts express TWEAK receptor fibroblast growth factor-inducible 14. In addition, TWEAK enhanced in vitro osteoclastogenesis without the presence of RANKL-providing cells and by inducing RANKL expression in fibroblast-like synoviocytes. Moreover, treatment with fibroblast growth factor-inducible 14-Fc inhibited RANKL-induced osteoclastogenesis, indicating that endogenous TWEAK also has osteoclastogenic activity. Our data demonstrated that TWEAK promotes osteoclastogenesis in RA, suggesting that therapeutic strategies targeting TWEAK could be effective for treatment of patients with RA, especially in preventing bone destruction. (Am J Pathol 2013, 183: 857-867; http://dx.doi.org/10.1016/j.ajpath.2013.05.027)
\end{abstract}

Rheumatoid arthritis (RA) is the prototypical systemic autoimmune disease with unknown etiology. RA is characterized by a hyperplastic synovial membrane that is capable of destroying the adjacent articular cartilage and bone. ${ }^{1,2}$ Among various pathological events in rheumatoid joints, bone destruction is clinically critically important because it is closely associated with RA patient disability, and the progression of joint damage can occur in patients with RA who are in prolonged remission. ${ }^{3}$ Osteoclasts, specialized bone-resorbing cells regulated by receptor activator of NF$\kappa \mathrm{B}$ ligand (RANKL) and macrophage colony-stimulating factor (M-CSF), are the leading player of bony erosion in RA. ${ }^{4,5}$ In the microenvironment of inflamed RA joints, local production of proinflammatory cytokines [IL-1, tumor necrosis factor (TNF)- $\alpha$, IL-6, and IL-17] and RANKL, provided by activated $\mathrm{CD} 4^{+} \mathrm{T}$ cells and synoviocytes, leads to stimulation of osteoclastogenesis and bone destruction. ${ }^{6,7}$
TNF-like weak inducer of apoptosis (TWEAK; TNFSF12) is a protein that, in humans, is encoded by the TNFSF 12 gene. It is a member of the TNF family that was originally identified as a weak inducer of apoptosis. ${ }^{8}$ In addition to stimulation of apoptosis, TWEAK has diverse biological activities, including stimulation of cell proliferation, angiogenesis, and induction of proinflammatory cytokines (chemokines). ${ }^{9-11}$ Fibroblast growth factor-inducible 14 (Fn14; TNFRSF12A), a protein that, in humans, is encoded by the TNFRSF $12 A$ gene, has been identified to be a TWEAK receptor and is widely expressed on

\footnotetext{
Supported by the Basic Science Research Program through the National Research Foundation of Korea, funded by the Ministry of Education, Science and Technology grant 20008-2005645 (to M.-L.C.) and by Korea Health Technology R\&D Project, Ministry for Health, Welfare \& Family Affairs, Republic of Korea grant A092258 (to M.-L.C.).

J.-S.P. and S.-K.K. contributed equally to this work.

Y.-G.C. and M.-L.C. contributed equally to this work as senior authors.
} 
various tissues. ${ }^{11,12}$ Several studies have reported direct and indirect evidence showing TWEAK might be implicated in the pathogenesis of RA. ${ }^{13-19}$ TWEAK has been shown to increase the production of proinflammatory cytokines, such as prostaglandin E2, matrix metalloproteinase (MMP)-1, and IL-6, in cultured fibroblast-like synoviocytes (FLSs) from patients with RA. ${ }^{13,16}$ TWEAK also promotes the proliferation of FLSs from patients with RA. ${ }^{16}$ TWEAK is highly expressed in synovial tissues of patients with RA compared with patients with osteoarthritis, ${ }^{18,19}$ and serum levels of TWEAK are increased and well correlated with disease activity in patients with RA. ${ }^{17}$ Two separate groups reported that blocking TWEAK with a neutralizing monoclonal antibody significantly reduces the clinical severity of collagen-induced arthritis (CIA) in a well-established murine model of RA, ${ }^{14,15}$ further supporting the relationship between TWEAK and RA. However, the exact role of TWEAK on osteoclastogenesis has not yet been fully determined.

The aim of the present study was to examine whether TWEAK has an osteoclastogenic impact in RA. We investigated the expression of TWEAK in the synovium, serum, and synovial fluid (SF) of patients with RA and in the synovium of animal models of RA, including CIA mice and IL-1 receptor antagonist knockout (IL-1RaKO) mice. We also examined whether TWEAK would induce expression of RANKL, the leading player of osteoclastogenesis ${ }^{20}$ in splenocytes from CIA mice, IL-1RaKO mice, and FLSs from patients with RA. We studied the osteoclastogenic potential of TWEAK on osteoclast precursor cells derived from human monocytes and on osteoclast precursor cells derived from mouse bone marrow-derived monocyte/macrophage (BMM) cells using tartrate-resistant acid phosphatase (TRAP) staining for osteoclasts. Our results indicate that TWEAK promotes osteoclastogenesis, suggesting that a strategy targeting TWEAK might be effective in treating patients with RA, especially for preventing bone destruction.

\section{Materials and Methods}

\section{Animals}

Male DBA/1J mice (aged 4 to 6 weeks) were purchased from SLC, Inc. (Shizuoka, Japan). IL-1RaKO mice were kindly obtained from Prof. Yoichiro Iwakura (Center for Experimental Medicine, Institute of Medical Science, University of Tokyo, Tokyo, Japan). They were maintained under specific pathogen-free conditions at the Institute of Medical Science, The Catholic University of Korea (Seoul) and were fed standard mouse chow (Ralston Purina, St. Louis, MO) and water ad libitum. All experimental procedures were examined and approved by the Animal Research Ethics Committee of The Catholic University of Korea.

\section{Induction of CIA}

To induce CIA in mice, $0.1 \mathrm{~mL}$ of an emulsion containing $100 \mu \mathrm{g}$ bovine type II collagen and complete Freund's adjuvant (Arthrogen-CIA; Chondrex, Seattle, WA) was injected intradermally into the base of the tail, as a primary immunization. Two weeks later, $100 \mu \mathrm{g}$ type II collagen, dissolved and emulsified 1:1 with incomplete Freund's adjuvant (Difco, Detroit, MI), was administered to the hind leg as a booster injection.

\section{IHC Data}

Mouse joint tissue and RA synovium were fixed in $4 \%$ paraformaldehyde, decalcified in 10\% EDTA for 2 to 3 weeks, trimmed, embedded in paraffin, and divided into sections. ${ }^{21}$ The sections were stained with H\&E, safranin O, and toluidine blue to detect proteoglycans. Immunostaining for TWEAK, Fn14, RANK, and RANKL was performed. Antibodies against mouse TWEAK, Fn14, RANK, and RANKL were obtained (Santa Cruz Biotechnology Inc., Santa Cruz, CA) and diluted to a $1-\mu \mathrm{g} / \mathrm{mL}$ concentration for TWEAK, Fn14, RANK, and RANKL. The same procedure was used for immunostaining of Fn14 in mature osteoclasts. The sections were counterstained with hematoxylin. Samples were imaged with a photomicroscope (Olympus, Tokyo).

\section{Preparation of Mixed Arthritic Joint Cells and Immunostaining for RANKL}

The ankle joints from 6- to 10-week-old normal, CIA, and IL1 RaKO mice were skinned and digested with $0.1 \mathrm{mg} / \mathrm{mL}$ liberase and $1 \mathrm{mg} / \mathrm{mL}$ DNase I (Roche Diagnostics Ltd, Burgess Hill, UK) for 60 minutes at $37^{\circ} \mathrm{C}$. Next, a $70-\mu$ m nylon cell strainer (BD Falcon, San Jose, CA) was used to process the digested tissue. The mixed joint cells were incubated in RPMI 1640 medium containing 5\% fetal bovine serum (FBS; Gibco, Carlsbad, CA) for 72 hours in the presence of TWEAK. After 72 hours, the mixed joint cells were attached to slides using a cytospin cytocentrifuge (Shandon Southern Products Ltd, Runcorn, Cheshire, UK) and fixed in $4 \%$ paraformaldehyde for 20 minutes at room temperature. The slides were incubated overnight at $4^{\circ} \mathrm{C}$ with primary antibodies directed against RANKL (Santa Cruz Biotechnology Inc.). The slides were washed for 15 minutes, followed by a 40-minute incubation with biotinylated secondary antibodies (Vector Laboratories, Burlingame, CA), and then treated with avidin-peroxidase complex for 1 hour at room temperature and visualized with 3,3-diaminobenzidine (DakoCytomation, Carpinteria, CA) staining. The slides were counterstained with hematoxylin.

\section{Isolation of Splenocytes and $\mathrm{CD}^{+}{ }^{+} \mathrm{T}$ Cells}

Mouse spleens were sieved through a mesh, and red blood cells were lysed using hypotonic ammonium-chloridepotassium buffer $\left[0.15 \mathrm{mmol} / \mathrm{L} \mathrm{NH}_{4} \mathrm{Cl}, 1 \mathrm{mmol} / \mathrm{L} \mathrm{KCO}{ }_{3}\right.$, and $0.1 \mathrm{mmol} / \mathrm{L}$ EDTA (pH 7.4)]. The remaining splenocytes were maintained in RPMI 1640 medium containing 5\% FBS. To purify splenic $\mathrm{CD} 4^{+} \mathrm{T}$ cells, the splenocytes were incubated with CD4-coated magnetic beads and isolated on 
MACS columns for cell separation (Miltenyi Biotec, Bergisch Gladbach, Germany).

\section{Mouse in Vitro 0steoclastogenesis}

Bone marrow cells (BMCs) were isolated from the tibias and femurs of mice by flushing the bone marrow cavity with $\alpha$ modified Eagle's medium (MEM; Invitrogen, Carlsbad, CA). The cells were centrifuged, exposed to ACK buffer at room temperature for 30 seconds to remove red blood cells, and incubated with $\alpha$-MEM containing penicillin/streptomycin and $10 \%$ FBS for 12 hours to separate the floating and adherent cells. The floating cells were collected, suspended in $\alpha$-MEM, counted, seeded in 48 -well plates at $1 \times 10^{5}$ cells per well, and cultured with $\alpha$-MEM in the presence of $10 \mathrm{ng} / \mathrm{mL}$ recombinant human M-CSF (R\&D Systems Minneapolis, MN) for 3 days to form macrophage-like osteoclast precursor cells. After 3 days, nonadherent cells were washed out and adherent cells were used as BMM cells. These osteoclast precursor cells were then cultured in the presence of $10 \mathrm{ng} / \mathrm{mL}$ recombinant human M-CSF, $25 \mathrm{ng} / \mathrm{mL}$ soluble recombinant human RANKL (PeproTech, London, UK), and $0.1 \mathrm{ng} / \mathrm{mL}$ recombinant mouse TWEAK for 4 days to generate osteoclasts.

\section{Flow Cytometry}

Osteoclast precursor cells from mice and humans were washed out with PBS, and the adherent cells were detached using TrypLE Express (Invitrogen, Carlsbad, CA). These cells were surface labeled with anti-Fn14 antibody (eBioscience, San Diego, CA) for 30 minutes before being subjected to FACS Calibur (BD Bioscience, San Jose, CA). Data were analyzed with FlowJo software version 8.6.3 (Tree Star, Ashland, OR).

\section{Confocal Microscopy of Immunostaining}

Osteoclast precursor cells from mice and humans were washed out with PBS, and cells were air dried and fixed with methanol, blocked with $10 \%$ goat serum for 30 minutes at room temperature, and then stained for Fn14 overnight at $4^{\circ} \mathrm{C}$. Confocal images were acquired using an LSM 510 confocal microscope (Zeiss, Oberkochen, Germany).

\section{Patients}

Patients with RA were studied in this investigation, and all patients met the American College of Rheumatology 1987 revised criteria for RA classification. ${ }^{22} \mathrm{SF}$ and serum were obtained from patients with RA. Patients with OA were studied as controls. Informed consent was obtained from all patients and healthy volunteers before the study, and the study was approved by the Seoul St. Mary's Hospital Ethics Committee.

\section{FLS Isolation}

FLSs from patients with RA were obtained as described previously, ${ }^{23}$ and FLSs from passages 4 to 8 were used for the experiments. The cells were stimulated with various concentrations of recombinant human TWEAK $(0.1,1$, or 5 $\mathrm{ng} / \mathrm{mL}$ ) for 72 hours.

\section{Human in Vitro Osteoclastogenesis}

Peripheral blood mononuclear cells obtained from normal healthy human volunteers were separated from buffy coats by Ficoll-Hypaque (Amersham Biosciences, Uppsala, Sweden) density gradient centrifugation. Removed red blood cells were seeded in 24 -well plates at $5 \times 10^{5}$ cells per well and incubated at $37^{\circ} \mathrm{C}$ for 2 hours to separate floating and adherent cells. Nonadherent cells were removed, and adherent cells were washed with sterile PBS and cultured in $\alpha$-MEM with $100 \mathrm{ng} / \mathrm{mL}$ recombinant human M-CSF for 3 days to form osteoclast precursor cells. After 3 days, these osteoclast precursor cells were cultured further in the presence of $25 \mathrm{ng} / \mathrm{mL}$ recombinant human M-CSF, $30 \mathrm{ng} / \mathrm{ml}$ recombinant human RANKL, and 1 or 10 $\mu \mathrm{g} / \mathrm{mL}$ recombinant human TWEAK or Fn14-Fc (R\&D Systems) for 9 days to generate osteoclasts. On day 3, the medium was replaced with a fresh medium containing M-CSF, RANKL, and TWEAK.

\section{Co-Culture System of Human Peripheral Blood Mononuclear Cells and FLSs}

FLSs from patients with RA in passages 4 to 8 were seeded in 60-mm dishes at $1 \times 10^{5}$ cells per dish, with Dulbecco's MEM containing 10\% FBS overnight to attach to the well, and the cells were serum starved with $1 \times$ insulintransferrin-selenium A (Invitrogen) for 12 hours. The cells were washed with PBS and then stimulated with various concentrations of TWEAK for 2 days, after which the cells were detached using trypsin-EDTA, and the medium was changed to $\alpha$-MEM containing $10 \%$ FBS. A total of $5 \times 10^{2}$ FLS cells per well were co-cultured with human osteoclast precursor cells, which had been prepared in 12-well plates, with $25 \mathrm{ng} / \mathrm{mL}$ M-CSF and $10 \mathrm{ng} / \mathrm{mL}$ RANKL. On day 3, the medium was replaced with a fresh medium containing M-CSF and RANKL.

\section{TRAP Staining}

A commercial TRAP kit (Sigma-Aldrich, St. Louis, MO) was used according to the manufacturer's instructions.

\section{Fn14 shRNA Transfection}

THP-1 cells were transfected with $1 \mu \mathrm{g}$ Fn14 shRNA or control shRNA plasmid (Santa Cruz Biotechnology Inc.) using Lipofectamine 2000 (Invitrogen), in accordance with the manufacturer's instructions. To monitor the transfection efficiency, green fluorescent protein expression vector was transfected into cells in parallel in all transfections, and at least $80 \%$ transfection efficiency was achieved. 
Gene Expression Analysis by Real-Time PCR

PCR amplification and analysis were achieved using a LightCycler 2.0 instrument (Roche Diagnostic, Mannheim, Germany) with software, version 4.0. All reactions were performed with the LightCycler FastStart DNA Master SYBR Green I (Takara, Shiga, Japan), according to the manufacturer's instructions. The following primers for human samples were used: TWEAK, 5'-CCCTGCGCTGCCTGGAGGAA-3' (sense) and 5'-AGACCAGGGCCCCTCAGTGA-3' (antisense); RANKL, 5'-ACCAGCATCAAAATCCCAAG-3' (sense) and 5'-CCCCAAAGTATGTTGCATCC- $3^{\prime}$ (antisense); cathepsin K, 5'-TGAGGCTTCTCTTGGTGTCCATAC- $3^{\prime}$ (sense) and $5^{\prime}$-AAAGGGTGTCATTACTGCGGG-3' (antisense); MMP-9, 5'-CGCAGACATCGTCATCCAGT-3' (sense) and 5'-GGATTGGCCTTGGAAGATGA-3' (antisense); calcitonin receptor, $5^{\prime}$-TGGTGCCAACCACTATCCATGC- $3^{\prime}$ (sense) and 5'CACAAGTGCCGCCATGACAG- $3^{\prime}$ (antisense); RANK, $5^{\prime}$-GCTCTAACAAATGTGAACCAGGA-3' (sense) and $5^{\prime}$-GCCTTGCCTGTATCACAAACT-3' (antisense); TRAP, 5'-GACCACCTTGGCAATGTCTCTG-3' (sense) and 5'TGGCTGAGGAAGTCTCTGAGTTG- $3^{\prime}$ (antisense); and $\beta$-actin, $5^{\prime}$-GGACTTCGAGCAAGAGATGG- $3^{\prime}$ (sense) and $5^{\prime}$-TGTGTTGGCGTACAGGTCTTTG- $3^{\prime}$ (antisense). The following primers for mouse samples were used: calcitonin receptor, 5'-CGGACTTTGACACAGCAGAA-3' (sense) and 5'-AGCAGCAATCGACAAGGAGT-3' (antisense); carbonic anhydrase II, 5'-TGGTTCACTGGAACACCAAA- $3^{\prime}$ (sense) and 5'-AGCAAGGGTCGAAGTTAGCA-3' (antisense); cathepsin K, 5'-CAGCAGAGGTGTGTACTATG- $3^{\prime}$ (sense) and 5'-GCGTTGTTCTTATTCCGAGC-3' (antisense); MMP-9, 5'-CTGTCCAGACCAAGGGTACAGCCT- $3^{\prime}$ (sense) and $5^{\prime}$-GAGGTATAGTGGGACACATAGTGG- $3^{\prime}$ (antisense); RANK, 5'-CGAGGAAGATTCCCACAGAG-3' (sense) and 5'-CAGTGAAGTCACAGCCCTCA- $3^{\prime}$ (antisense); vascular endothelial growth factor (VEGF), 5'-TCTTCAAGCCGTCCTGTGTG-3' (sense) and $5^{\prime}$-AGGACCATTTACACGTCTGC- $3^{\prime}$ (antisense); IL-1 $\beta, \quad 5^{\prime}$-GGATGAGGACATGAGCACATTC- ${ }^{\prime}$ (sense) and $5^{\prime}$-GGAAGACAGGCTTGTGCTCTGA-3' (antisense); TNF- $\alpha, 5^{\prime}$-ATGAGCACAGAAAGCATGATC-3' (sense) and $5^{\prime}$-TACAGGCTTGTCACTCGAATT-3' (antisense); and $\beta$-actin, $5^{\prime}$-GTACGACCAGAGGCATACAGG- $3^{\prime}$ (sense) and 5'-GATGACGATATCGCTGCGCTG-3' (antisense). The level of mRNA expression was normalized to that of $\beta$-actin.

\section{ELISA}

Production of RANKL and TWEAK was measured with ELISA kits for human soluble RANKL and TWEAK (both from PeproTech, Rocky Hill, NJ), according to the manufacturer's instructions. The amounts of IL- $1 \beta$, TNF- $\alpha$, and VEGF in the culture supernatants from CIA splenocytes were measured by sandwich ELISA (R\&D Systems). The absorbance was measured at $405 \mathrm{~nm}$ on an ELISA microplate reader (Molecular Devices, Sunnyvale, CA).

\section{Statistical Analysis}

Statistical analyses were performed using SAS software, version 9 (SAS Institute, Inc., Cary, NC). Experimental values are presented as means $\pm \mathrm{SD}$. Comparisons of numerical data between two groups were performed by Student's $t$-test or $U$ test. Differences in the mean values of various groups were analyzed using analysis of variance with a post hoc test. $P<$ 0.05 (two tailed) was considered significant.

\section{Results}

\section{TWEAK Promotes 0steoclastogenesis in Mice}

We first examined whether osteoclast precursor cells or osteoclasts contain the functional TWEAK receptor, Fn14, in mice. BMCs obtained from C57BL/6 mice were cultured with $10 \mathrm{ng} / \mathrm{mL}$ M-CSF for 72 hours to form osteoclast precursor cells. The expression of Fn14 was determined by flow cytometry and confocal microscopy. The results showed that treatment with M-CSF increased expression of Fn14 in a time-dependent manner. The additional treatment with $100 \mathrm{ng} / \mathrm{mL}$ RANKL did not further increase the expression of Fn14 (Figure 1, A and B).

Our next experiment was to examine whether TWEAK has osteoclastogenic impact. Osteoclast precursor cells derived from mouse BMM cells were stimulated with TWEAK, M-CSF, and RANKL to induce osteoclastogenesis. TWEAK alone failed to induce osteoclastogenesis, as did M-CSF (data not shown). However, to our surprise, TWEAK promoted osteoclastogenesis in the presence of M-CSF and a low dose of RANKL $(10 \mathrm{ng} / \mathrm{mL}$ ) (Figure 1C). These findings were evaluated by counting TRAP-positive cell numbers per well. The expression levels of various osteoclastogenic markers, such as cathepsin K, MMP-9, carbonic anhydrase II, calcitonin receptor, and RANK, were also measured by real-time PCR (Figure 1D). TWEAK increased the expression of various osteoclastogenic markers in the presence of M-CSF and low-dose RANKL $(10 \mathrm{ng} / \mathrm{mL})$. We also demonstrated that treatment with TWEAK increased the expression of osteoclastogenic markers in splenocytes from normal mice (Figure 1E).

TWEAK Enhances RANKL Expression in Mixed Joint Cells and Splenocytes from Mice

Because RANKL is known to be the prototypical mediator of osteoclastogenesis, ${ }^{24}$ we investigated whether TWEAK could induce expression of RANKL in vitro. We isolated mixed joint cells from normal DBA/1J mice and two animal models of RA (CIA and IL-1RaKO mice). We then added TWEAK to the mixed joint cell cultures and analyzed the expression of RANKL by immunostaining. As shown in Figure 2A, TWEAK profoundly increased the expression of RANKL in mixed joint cells. Treatment with TWEAK also 
A
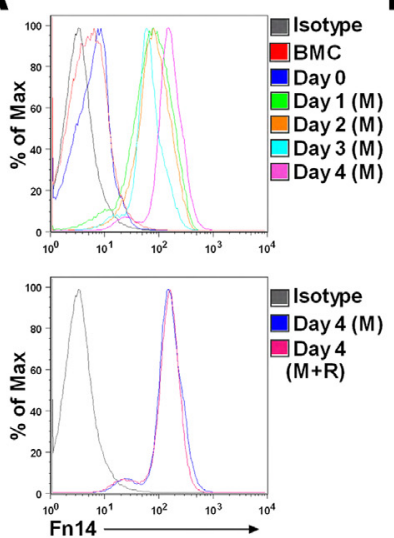

C
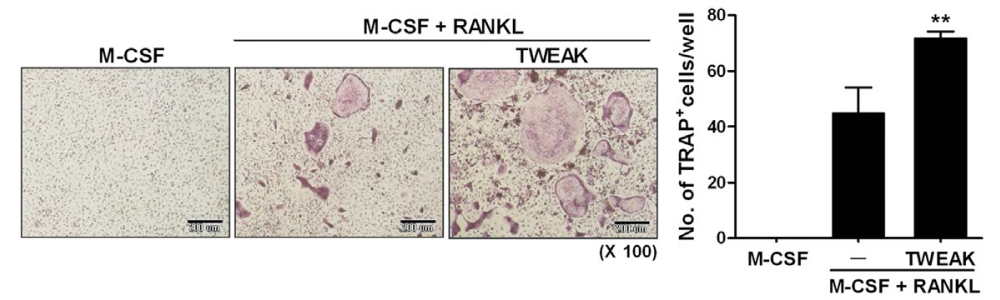

D
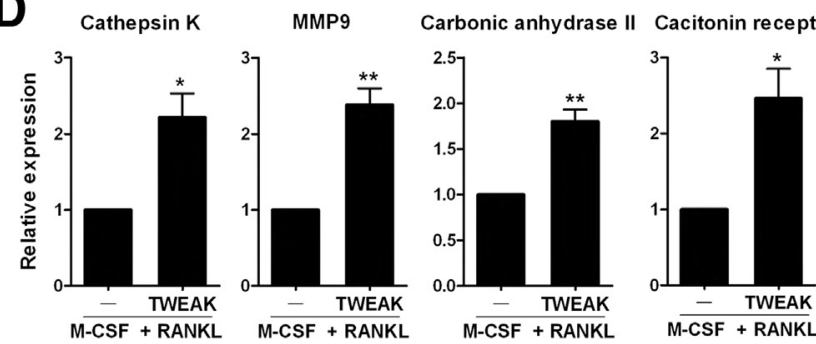

E
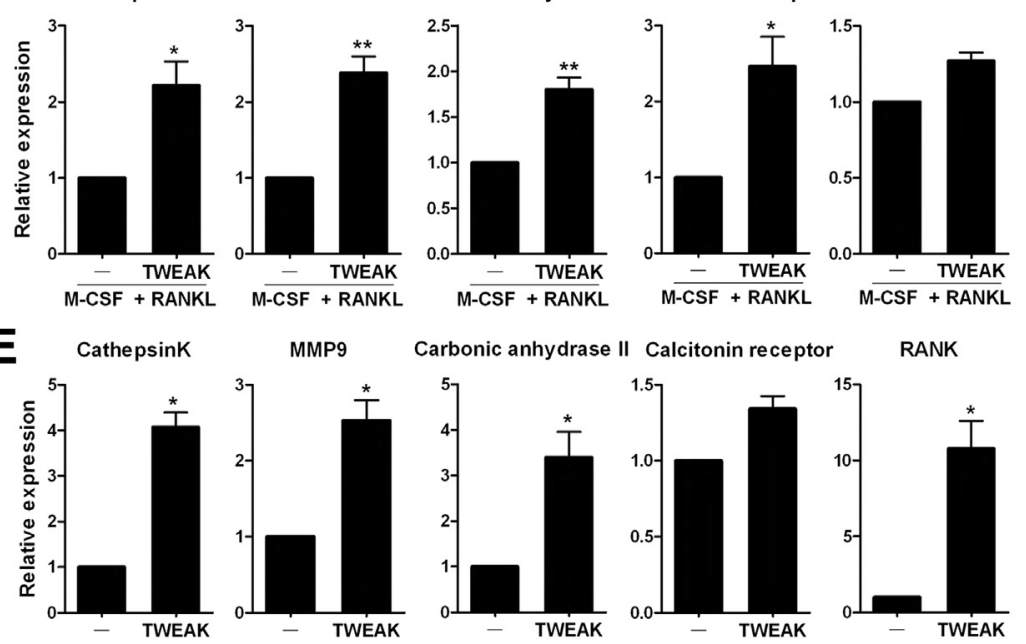

RANK

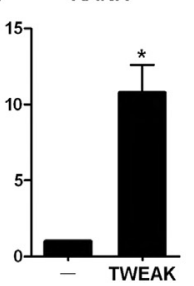

Figure 1 TWEAK promotes osteoclastogenesis in mice. A and B: Cell surface expression of Fn14 on osteoclast precursor cells in mice. BMCs were isolated from $\mathrm{C} 57 \mathrm{BL} / 6$ mice and cultured with 10 $\mathrm{ng} / \mathrm{mL}$ M-CSF for 72 hours to form osteoclast precursor cells. The time course of Fn14 expression was determined by flow cytometry (A) and confocal microscopy (B). Fn14-expressing cells are shown in red. DAPI staining for nuclei is shown as blue. $M, 10 \mathrm{ng} / \mathrm{mL}$ M-CSF; Max, maximum; R, 100 $\mathrm{ng} / \mathrm{mL}$ RANKL. C: TWEAK-promotes osteoclast formation in the presence of M-CSF and low-dose RANKL (10 $\mathrm{ng} / \mathrm{mL})$. DBA/1J mouse osteoclast precursor cells were further cultured in the presence of $10 \mathrm{ng} / \mathrm{mL} M-C S F$ and $10 \mathrm{ng} / \mathrm{mL}$ RANKL and/ or $0.1 \mathrm{ng} / \mathrm{mL}$ TWEAK for 4 days. The cells were stained for TRAP activity, and multinucleated TRAP-positive cells were counted as osteoclasts. D: mRNA levels of cathepsin K, MMP-9, carbonic anhydrase II, calcitonin receptor, and RANK were quantified by real-time PCR. ${ }^{*} P<0.05,{ }^{*} * P<$ 0.01 compared with the M-CSF- and RANKLtreated cells (C and D). E: TWEAK induces the expression of various osteoclastogenic markers in splenocytes from DBA/1J mice. Splenocytes from normal mice were cultured with or without $25 \mathrm{ng} /$ $\mathrm{mL}$ TWEAK for 72 hours. mRNA levels of cathepsin K, MMP-9, carbonic anhydrase II, calcitonin receptor, and RANK were quantified by real-time PCR. ${ }^{*} P<0.05$ compared with untreated cells (E). Original magnification: $\times 1000(B) ; \times 100(C)$. increased mRNA levels of RANKL in splenocytes from normal DBA/1J, CIA, wild-type (WT; C57BL/6), and IL$1 \mathrm{RaKO}$ mice (Figure 2B), determined by real-time PCR. To investigate the effect of TWEAK on the production of cytokines, which are implicated in the pathogenesis of RA, we cultured splenocytes from CIA mice with TWEAK. We then measured IL- $1 \beta$, TNF- $\alpha$, and VEGF. The results showed that TWEAK markedly up-regulated the production of IL-1 $\beta$, TNF- $\alpha$, and VEGF in splenocytes from CIA mice (Figure 2C).

TWEAK Is Highly Expressed in Joint Tissues of Animal Models of RA (CIA and IL-1RaKO Mice) and Patients with RA

To verify the observation that TWEAK induces RANKL expression in mixed joint cells in vitro, we performed immunohistochemical (IHC) staining to investigate the distribution and localization of TWEAK and Fn14 in joint tissues from CIA, IL-1RaKO, and normal mice. As shown in Figure 2D, increased expressions of TWEAK were observed in all of the synovia of CIA and IL1 RaKO mice. Positive staining was mainly observed in the pannus of the hyperplastic synovium. However, TWEAK was rarely expressed in the joints of normal mice. The IHC staining for Fn14, RANKL, and RANK was also performed on the joint tissues from CIA, IL$1 \mathrm{RaKO}$, and normal mice. The results showed that increased expressions of Fn14, RANKL, and RANK were also observed in the inflamed synovium of CIA and IL$1 \mathrm{RaKO}$ mice, as with TWEAK.

We also performed IHC staining of synovial tissues from patients with RA using antibodies to TWEAK, Fn14, RANK, and RANKL (Figure 3A). TWEAK was highly 

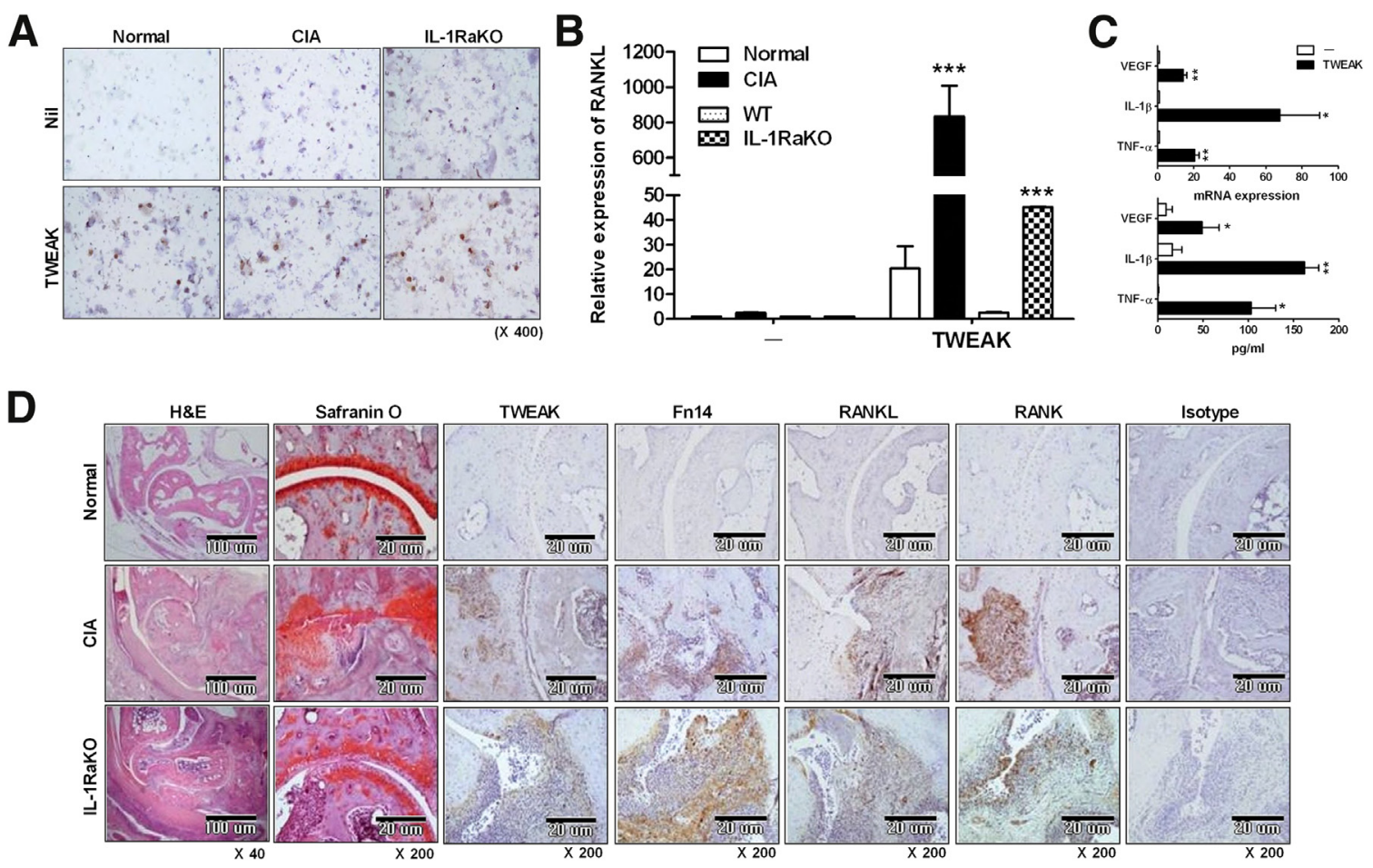

Figure 2 Increased expression of TWEAK in joint tissues from two animal models of RA. A: Treatment with TWEAK increases RANKL expression in mixed joint cells. Mixed joint cells from normal, CIA, and IL-1RaKO mice were cultured with or without $25 \mathrm{ng} / \mathrm{mL}$ TWEAK for 72 hours. Cells were immunostained with anti-TWEAK antibodies. Original magnification, $\times 400$. Cells stained with antibodies are shown in brown. A representative result is shown. B: Treatment with TWEAK increases RANKL expression in splenocytes. Splenocytes from normal DBA/1J, CIA, WT (C57BL/6 mice), and IL-1RaK0 mice were cultured with or without $25 \mathrm{ng} / \mathrm{mL}$ TWEAK for 72 hours. mRNA levels of RANKL were determined by real-time PCR. ${ }^{* * *} P<0.001$ compared with normal or WT. C: TWEAK enhances the production of IL-1 $\beta$, TNF- $\alpha$, and VEGF in splenocytes. Splenocytes from CIA mice were cultured with or without $25 \mathrm{ng} / \mathrm{mL}$ TWEAK for 24 or 72 hours. After 24 hours, mRNA levels of IL-1 $\beta$, TNF- $\alpha$, and VEGF were determined by real-time PCR. After 72 hours, concentrations of IL-1 $\beta$, TNF- $\alpha$, and VEGF in the culture supernatant were measured by ELISA. Data are expressed as means \pm SD. ${ }^{*} P<0.05,{ }^{*} P<$ 0.01 compared with Nil. D: TWEAK, Fn14, RANKL, and RANK are highly expressed in joint tissues from CIA and IL-1RaK0 mice. Tissue sections from the joints of control mice $(n=3)$, CIA mice $(n=3)$, and IL-1RaKO mice $(n=3)$ were stained with H\&E and safranin 0 . Tissue sections from the joints of control mice $(n=3)$, CIA mice $(n=3)$, and WT mice $(n=3)$ were stained with anti-TWEAK, anti-Fn14, anti-RANKL, and anti-RANK antibodies. Cells stained with each antibody are shown in brown.

expressed in all synovial tissue sections from patients with RA, as with Fn14, RANK, and RANKL. However, TWEAK was scarcely expressed in synovial tissues from patients with OA.

In addition, we measured serum and SF levels of TWEAK and soluble RANKL in patients with RA $(n=28)$ and patients with OA $(n=28)$. Compared with patients with OA, patients with RA had significantly higher serum and SF concentrations of TWEAK [serum TWEAK, $3571.70 \pm$ 2566.43 versus $2105.42 \pm 166.23 \mathrm{pg} / \mathrm{mL}(P<0.05)$; SF TWEAK, $31,373.59 \pm 21,772.35$ versus $6274.23 \pm$ $10,070.26 \mathrm{pg} / \mathrm{mL}(P<0.001)]$. Patients with RA also had significantly higher serum and SF levels of soluble RANKL [serum RANKL, $713.36 \pm 903.87$ versus $304.25 \pm 450.14$ $\mathrm{pg} / \mathrm{mL}(P<0.05)$; SF RANKL, $1267.19 \pm 450.14$ versus $187.48 \pm 498.92 \mathrm{pg} / \mathrm{mL}(P<0.01)]$ (Figure 3B). In patients with RA, serum TWEAK levels showed a significantly positive correlation with serum-soluble RANKL levels $\left(R^{2}=0.8006, P<0.001\right)$. SF TWEAK levels also positively correlated with SF-soluble RANKL levels $\left(R^{2}=0.4464\right.$, $P<0.001$ ) (Figure 3C).

\section{TWEAK Promotes 0steoclastogenesis in Humans through Up-Regulation of RANKL in FLSs}

Rheumatoid synoviocytes are known to express RANKL and, thereby, promote osteoclastogenesis. ${ }^{25,26}$ Therefore, we investigated whether TWEAK could induce RANKL expression in synoviocytes from patients with RA. TWEAK increased RANKL expression in cultured RA-FLSs $(n=4)$, which was determined by real-time PCR (Figure 4A). TWEAK also induced the production of soluble RANKL in cultured RA-FLSs $(n=4)$ (Figure 4B). Therefore, it can be speculated that TWEAK has osteoclastogenic potential in inflamed joints of patients with RA because TWEAK induced RANKL expression in RA-FLSs.

To investigate the functional potency of TWEAKinduced RANKL expression by synoviocytes to induce osteoclast precursor cell differentiation into mature osteoclasts, we co-cultured TWEAK-stimulated or unstimulated synoviocytes with monocyte-derived osteoclast precursor cells. After 9 days of culture, multinucleated mature osteoclasts had differentiated from osteoclast precursor cells, as 

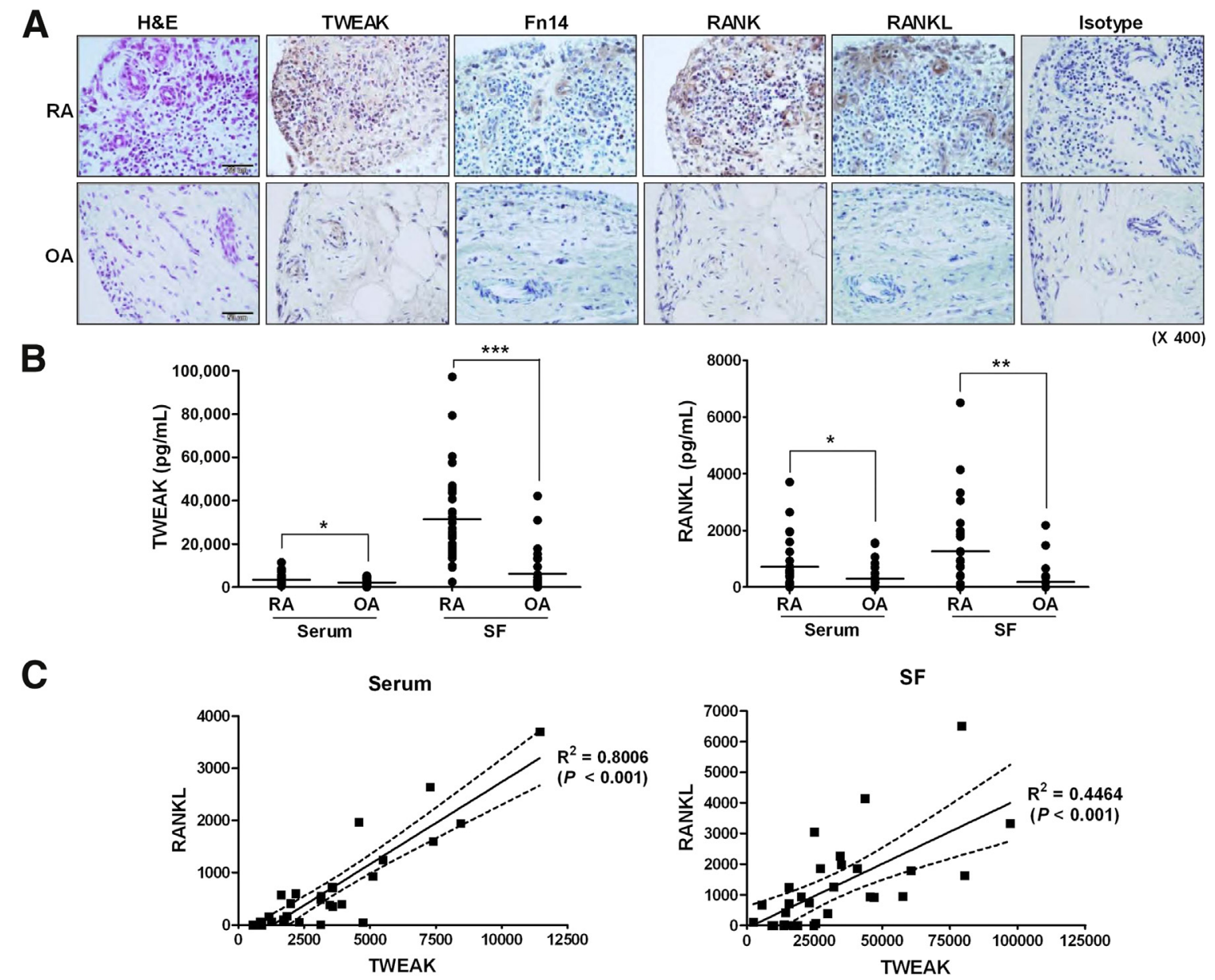

(400)

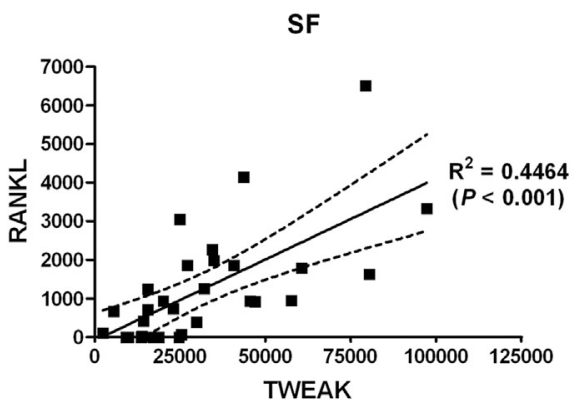

Figure 3 Increased expression of TWEAK in patients with RA. A: Tissue sections from the synovia of patients with RA $(n=3)$ or OA $(n=3)$ were stained with anti-TWEAK, anti-Fn14, anti-RANK, and anti-RANKL antibodies. Cells stained with each antibody are shown in brown. Representative results are shown. Original magnification, $\times 400$. B: Serum and SF levels of TWEAK and soluble RANKL are significantly higher in patients with RA compared with 0 A. Concentrations of TWEAK and soluble RANKL in the serum and SF of patients with RA $(n=28)$ or OA $(n=28)$ were determined using ELISA. * $P<0.05, * * P<0.01$, and ${ }^{* *} P<0.001$ compared with OA. C: Correlation of RANKL levels with TWEAK levels in patients with RA. There are positive correlations between serumsoluble RANKL and TWEAK levels $(P<0.001)$ and between SF RANKL and TWEAK levels $(P<0.001)$.

determined by the TRAP staining assay. As expected, TWEAK-stimulated synoviocytes were more potent than unstimulated synoviocytes in their osteoclastogenic potential (Figure 4, C and D). In addition, TWEAK-stimulated synoviocytes promote osteoclastogenesis in a TWEAK concentration-dependent manner.

\section{Endogenous TWEAK Promotes Differentiation of Human Osteoclast Precursor Cells into Mature Osteoclasts}

We investigated whether human osteoclast precursor cells or osteoclasts have functional $\mathrm{Fn} 14$, as found in mice. As shown in Figure 5A, both a confocal microscopy examination and flow cytometry analysis showed that Fn14 was significantly expressed on osteoclast precursor cells derived from human monocytes.

Our next experiment was to examine whether TWEAK has osteoclastogenic impact in humans without RANKL-providing cells, such as synoviocytes. Osteoclast precursor cells were prepared from patients with RA and then stimulated with TWEAK, M-CSF, and RANKL to induce osteoclastogenesis. TWEAK alone failed to induce osteoclastogenesis (data not shown), as did M-CSF. However, TWEAK profoundly promoted osteoclastogenesis in the presence of M-CSF and a low dose of RANKL $(10 \mathrm{ng} / \mathrm{mL})$. These findings were evaluated by counting TRAP-positive cell numbers per well (Figure 5B).

In an attempt to determine whether endogenous TWEAK contributes to osteoclastogenesis in patients with RA, we evaluated the expression of TWEAK in human osteoclast precursor cells. The result showed that in vitro treatment with $10 \mathrm{ng} / \mathrm{mL}$ M-CSF and $10 \mathrm{ng} / \mathrm{mL}$ RANKL up-regulated mRNA levels of TWEAK in human osteoclast precursor cells (Figure 5C). We indicated that endogenous TWEAK contributes to osteoclastogenesis by showing that treatment with Fn14-Fc attenuated osteoclastogenesis induced by MCSF and RANKL (Figure 5, D and E). We also performed 


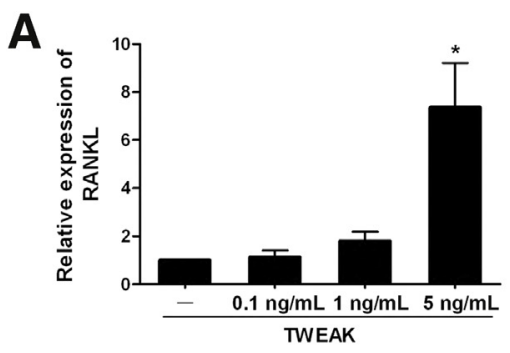

C
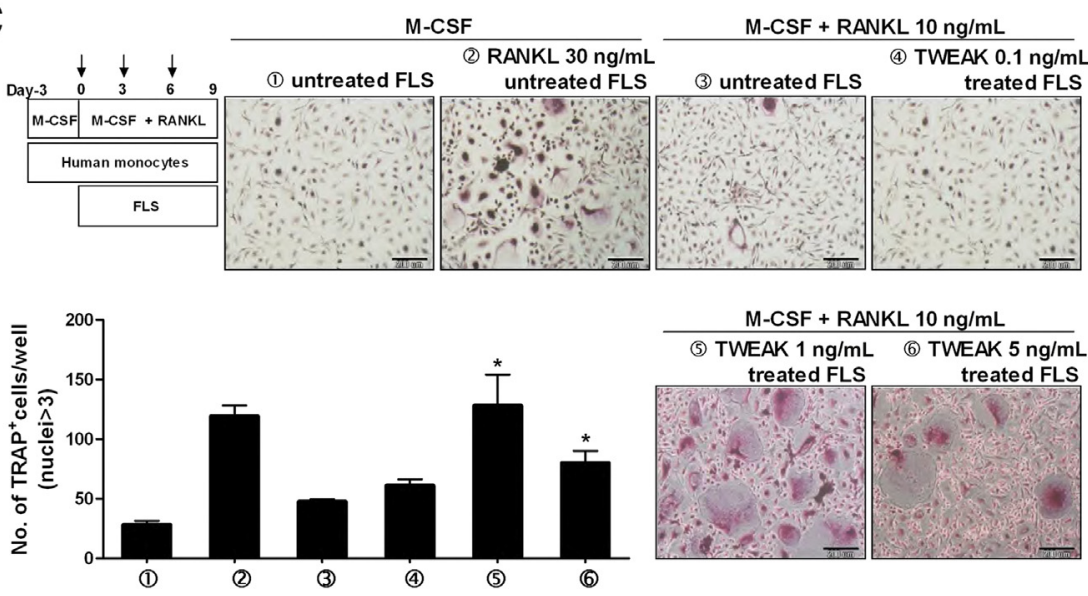

$\mathrm{M}-\mathrm{CSF}+\mathrm{RANKL} 10 \mathrm{ng} / \mathrm{mL}$

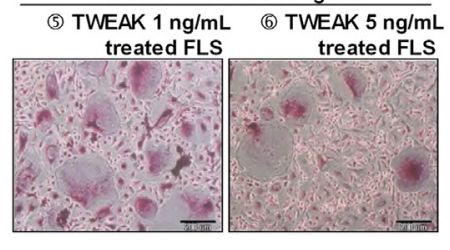

D
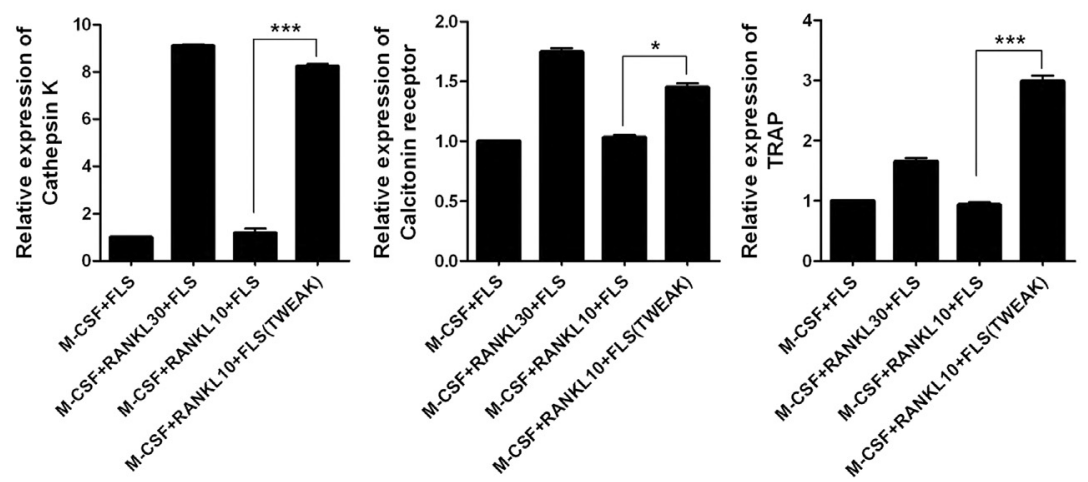

Figure 4 TWEAK promotes osteoclastogenesis through induction of RANKL in human synovial fibroblasts. A: Treatment with TWEAK $(0.1,1$, or 5 $\mathrm{ng} / \mathrm{mL}$ ) for 72 hours dose dependently increases RANKL expression in FLSs obtained from four patients with RA. Levels of mRNA of RANKL were determined by real-time PCR. B: Treatment with TWEAK $(0.1,1$, or $5 \mathrm{ng} / \mathrm{mL})$ for 72 hours dose dependently increases the production of soluble RANKL in FLSs obtained from four patients with RA. Levels of RANKL in culture supernatants were determined by ELISA. ${ }^{*} P<0.05(\mathrm{~A}),{ }^{* * *} P<$ 0.001 (B) compared with Nil. C: Co-culture of osteoclast precursor cells and TWEAK-stimulated FLSs from patients with RA show significantly more TRAP-positive osteoclasts. RA-FLSs were pretreated for 2 days with various concentrations of $\operatorname{TWEAK}(0.1,1$, or $5 \mathrm{ng} / \mathrm{mL})$; FLSs $\left(5 \times 10^{2}\right.$ cells per well) were co-cultured with osteoclast precursor cells from monocytes of healthy controls, which had been prepared in 12-well plates, and co-cultures were maintained for 9 days in the presence of $25 \mathrm{ng} / \mathrm{mL}$ M-CSF and RANKL $(0,10$, or $30 \mathrm{ng} / \mathrm{mL}$ ), as shown in the schematic diagram. The $30 \mathrm{ng} / \mathrm{mL}$ RANKL-stimulated condition served as the positive control. Cells were stained for TRAP activity, and the numbers of multinucleated TRAPpositive cells were determined. Original magnification, $\times 100 .{ }^{*} P<0.05$ versus the M-CSF, RANKL $(10 \mathrm{ng} / \mathrm{mL})$, and untreated FLS groups. D: mRNA levels of cathepsin $\mathrm{K}$, calcitonin receptor, and TRAP were quantified by real-time PCR. ${ }^{*} P<0.05$, $* * * P<0.001$ compared with the M-CSF, RANKL $(10 \mathrm{ng} / \mathrm{mL})$ with untreated $\mathrm{FLS}$. knockdown of Fn14 using shRNA in the monocytic THP-1 cells in the presence of $25 \mathrm{ng} / \mathrm{mL}$ M-CSF and/or $50 \mathrm{ng} / \mathrm{mL}$ RANKL. As shown in Figure 5F, knockdown of Fn14 decreased mRNA levels of TRAP in the presence of M-CSF and RANKL, further demonstrating that endogenous TWEAK also has osteoclastogenic activity.

\section{Discussion}

The present study was designed to investigate the effect of TWEAK on osteoclastogenesis in RA. We demonstrated that TWEAK is highly expressed in the synovium, serum, and SF of patients with RA, and these findings correspond well with results from earlier studies. ${ }^{17-19}$ We also showed increased expression of TWEAK in the synovium of two distinct animal models of RA, CIA and IL-1RaKO mice. Osteoclast precursor cells from both humans and mice contain functional TWEAK receptor, Fn14. Treatment with TWEAK increased the expression of RANKL in mixed joint cells and splenocytes from CIA mice, IL-1RaKO mice, and FLSs from patients with RA. We verified that TWEAK has osteoclastogenic potential in mice and humans.

Bone destruction is critically important for the prognosis of patients with RA because it is closely related to functional disability. Many drugs have been developed for the treatment of RA, most of which modulate immune reactions and reduce both inflammation and pain. Despite continuous treatment with conventional disease-modifying antirheumatic drugs, disease progression does not stop ${ }^{27,28}$ and some patients still have to undergo joint replacement surgery because of progressive bone destruction. ${ }^{29}$ Osteoclasts are specialized bone-resorbing cells, and they are the major cells implicated in bone destruction. Although several investigations have indicated that TWEAK might be implicated in the pathogenesis of $\mathrm{RA},{ }^{13-19}$ there have been few reports that examined the role of 

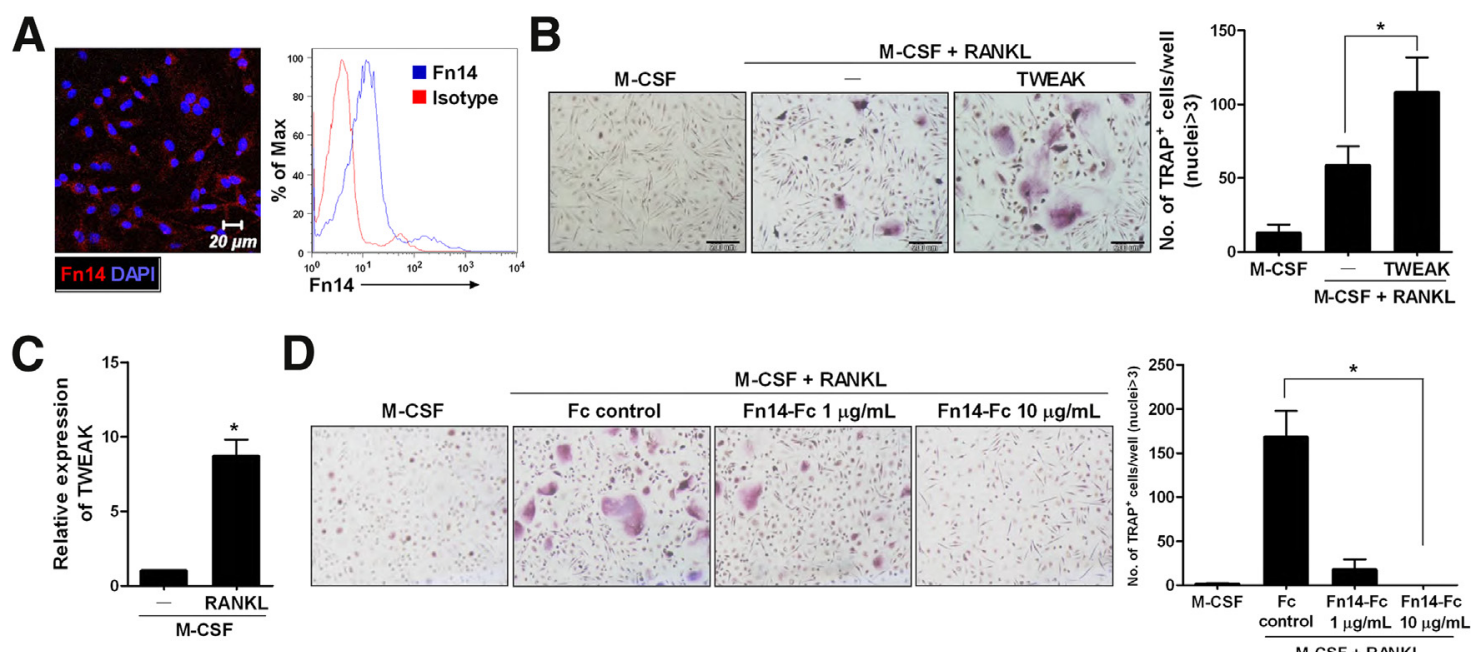

$\mathbf{E}$

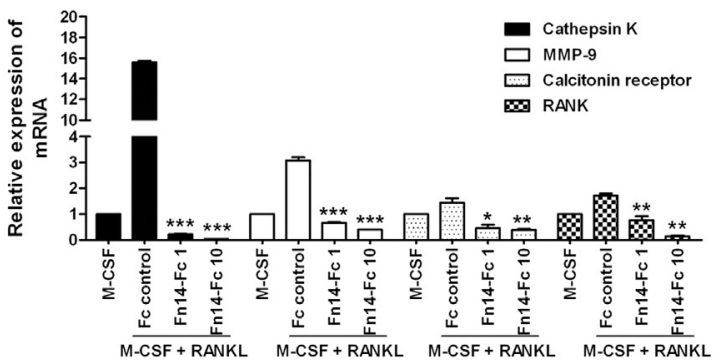

$\mathbf{F}$
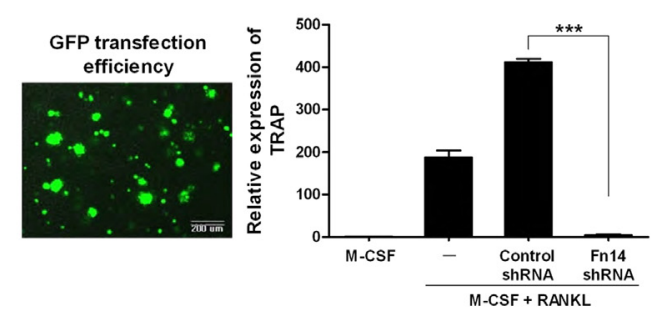

Figure 5 Endogenous TWEAK induced by M-CSF and RANKL promotes differentiation of human osteoclast precursor cells into osteoclasts. A: Cell surface expression of Fn14 on osteoclast precursor cells derived from human monocytes. The expression of Fn14 in osteoclast precursor cells was evaluated by confocal microscopy. Fn14-expressing cells are shown in red. DAPI staining for nuclei is shown in blue. 0steoclast precursor cells were stained with anti-Fn14 (blue line) or isotype control (red line) for 30 minutes and analyzed by flow cytometry. B: TWEAK-promotes differentiation of human osteoclast precursor cells into osteoclasts in the presence of M-CSF and low-dose RANKL $(10 \mathrm{ng} / \mathrm{mL})$. Human osteoclast precursor cells were cultured in the presence of $25 \mathrm{ng} / \mathrm{mL} \mathrm{M}$-CSF and/or $10 \mathrm{ng} / \mathrm{mL}$ RANKL and/or $1 \mathrm{ng} / \mathrm{mL}$ TWEAK for 9 days. The cells were stained for TRAP activity, and multinucleated TRAP-positive cells were counted as osteoclasts. C: Treatment with $25 \mathrm{ng} / \mathrm{mL}$ M-CSF and $30 \mathrm{ng} / \mathrm{mL}$ RANKL increases the expression of TWEAK in human osteoclast precursor cells. mRNA levels of TWEAK were quantified by real-time PCR. Representative results are shown. D: Treatment with Fn14-FC inhibits osteoclastogenesis induced by M-CSF and RANKL. Fn14-Fc (1 or $10 \mu \mathrm{g} / \mathrm{mL}$ ) was added to osteoclast precursor cells in the presence of $25 \mathrm{ng} / \mathrm{mL} \mathrm{M}$-CSF and $30 \mathrm{ng} / \mathrm{mL}$ RANKL, and the cells were incubated for 9 days to generate mature osteoclasts. The cells stained as multinucleated TRAP-positive cells were counted as osteoclasts. Original magnification, $\times 100$ (B and D). ${ }^{*} P<0.05$ compared with the M-CSF- and RANKL-treated cells (B and D). E: mRNA levels of cathepsin K, MMP-9, calcitonin receptor, and RANK were quantified by real-time PCR. ${ }^{*} P<0.05,{ }^{*} P<0.01$, and ${ }^{* * *} P<0.001$ compared with the Fc control-treated cells. F: Monocytic THP-1 cells were transfected with Fn14 shRNA or control shRNA plasmid, as described in Materials and Methods, and then cultured in the presence of $25 \mathrm{ng} / \mathrm{mL} \mathrm{M-CSF} \mathrm{and/or} 50 \mathrm{ng} / \mathrm{mL}$ RANKL for 24 hours. mRNA levels of TRAP were determined by real-time PCR. ${ }^{* * *} P<0.001$.

TWEAK on osteoclastogenesis. ${ }^{19,30}$ Polek et $\mathrm{al}^{30}$ demonstrated that TWEAK mediates the differentiation of RAW264.7 monocyte/macrophage into multinucleated, functional osteoclast in mice, consistent with our results showing that TWEAK promoted the differentiation of osteoclast precursor cells derived from mouse BMM cells into osteoclasts. Interestingly, Polek et $\mathrm{al}^{30}$ showed that the osteoclastogenic impact of TWEAK was direct and not mediated by RANKL. They also indicated that Fn14 was not responsible for the osteoclastogenic effect of TWEAK on RAW cells by showing that the expression of Fn14 was not observed in RAW cells. In contrast, our study demonstrated that both osteoclasts and osteoclast precursor cells express Fn14, the functional TWEAK receptor. In addition, TWEAK promoted osteoclastogenesis only in the presence of RANKL stimulation and TWEAK alone did not have direct osteoclastogenic impact. We are not able to fully explain the discordant results. One possible explanation is that osteoclast precursor cells, derived from BMM cells by treatment with M-CSF for 72 hours, express Fn14, whereas BMCs do not express Fn14 like RAW cells (data not shown). Thus, it could be speculated that M-CSF promoted the differentiation of BMM cells into osteoclast precursor cells and induced the expression of Fn14. Therefore, although one previous investigation demonstrated an RANKL- and Fn14-independent pathway of osteoclastogenesis, ${ }^{30}$ it is likely that TWEAK promotes osteoclastogenesis via Fn14 in the presence of RANKL.

Recently, Dharmapatni et $\mathrm{al}^{19}$ investigated TWEAK and Fn14 expression in the pathogenesis of joint inflammation and bone erosion in RA. They showed that TWEAK and Fn14 expressions are significantly higher in synovial tissues from patients with RA compared with synovial tissues from patients with OA, similar to our results. They also reported that 
TWEAK induces RANKL on osteoblasts, another RANKL providing cells such as synoviocytes. However, they described that M-CSF/RANKL-induced osteoclastogenesis was inhibited, rather than stimulated, by treatment with TWEAK, as assessed by both TRAP staining and bone resorption assay in humans and mice; this differs from our results demonstrating that TWEAK potentiates osteoclastogenesis induced by M-CSF/RANKL in humans and mice. Thus, there may be differences between the physiological osteoblast-mediated RANKL signaling and inflammatory situations in which the shedding of soluble RANKL may play a more prominent role. Osteoprotegerin might be implicated in this process. Comprehensive and intensive research is required to clarify these discordant results regarding the role of TWEAK in osteoclastogenesis. However, our results demonstrated that serum and SF TWEAK levels well correlated with soluble RANKL levels in patients with RA. We also showed that TWEAK induced the expression of RANKL in synoviocytes, the major effector cells in RA pathological characteristics, such as a previous report describing that TWEAK induces the expression of RANKL in osteoblasts. ${ }^{19}$ These findings are also indirect evidences supporting the osteoclastogenic potential of TWEAK. Therefore, we believe that TWEAK has osteoclastogenic effects.

One intriguing finding of this study is that recombinant exogenous TWEAK and endogenous TWEAK, produced by osteoclast precursor cells or osteoclasts, contribute to osteoclastogenesis in patients with RA. We found that treatment with M-CSF and RANKL induces the expression of TWEAK in human osteoclast precursor cells (Figure 5C). We finally demonstrated the osteoclastogenic effects of endogenous TWEAK, for the first time to our knowledge, by showing that treatment with Fn14-Fc attenuated osteoclastogenesis induced by M-CSF and RANKL (Figure 5D).

In conclusion, we have demonstrated that TWEAK promotes osteoclastogenesis in the absence of RANKLproviding cells and through up-regulation of RANKL expression in synoviocytes. These findings suggest that a novel therapy targeting TWEAK might be effective for preventing bone destruction in patients with RA.

\section{References}

1. Firestein GS: Invasive fibroblast-like synoviocytes in rheumatoid arthritis: passive responders or transformed aggressors? Arthritis Rheum 1996, 39:1781-1790

2. Feldmann M, Brennan FM, Maini RN: Rheumatoid arthritis. Cell 1996, 85:307-310

3. Molenaar ET, Voskuyl AE, Dinant HJ, Bezemer PD, Boers M, Dijkmans BA: Progression of radiologic damage in patients with rheumatoid arthritis in clinical remission. Arthritis Rheum 2004, 50:36-42

4. Gravallese EM: Bone destruction in arthritis. Ann Rheum Dis 2002, 61(Suppl 2):ii84-ii86

5. O'Gradaigh D, Ireland D, Bord S, Compston JE: Joint erosion in rheumatoid arthritis: interactions between tumour necrosis factor alpha, interleukin 1, and receptor activator of nuclear factor kappaB ligand (RANKL) regulate osteoclasts. Ann Rheum Dis 2004, 63:354-359

6. Lee SH, Kim TS, Choi Y, Lorenzo J: Osteoimmunology: cytokines and the skeletal system. BMB Rep 2008, 41:495-510
7. Lorenzo J, Horowitz M, Choi Y: Osteoimmunology: interactions of the bone and immune system. Endocr Rev 2008, 29:403-440

8. Chicheportiche Y, Bourdon PR, Xu H, Hsu YM, Scott H, Hession C, Garcia I, Browning JL: TWEAK, a new secreted ligand in the tumor necrosis factor family that weakly induces apoptosis. J Biol Chem 1997, 272:32401-32410

9. Lynch CN, Wang YC, Lund JK, Chen YW, Leal JA, Wiley SR: TWEAK induces angiogenesis and proliferation of endothelial cells. J Biol Chem 1999, 274:8455-8459

10. Wiley SR, Cassiano L, Lofton T, Davis-Smith T, Winkles JA, Lindner V, Liu H, Daniel TO, Smith CA, Fanslow WC: A novel TNF receptor family member binds TWEAK and is implicated in angiogenesis. Immunity 2001, 15:837-846

11. Wiley SR, Winkles JA: TWEAK, a member of the TNF superfamily, is a multifunctional cytokine that binds the TweakR/Fn14 receptor. Cytokine Growth Factor Rev 2003, 14:241-249

12. Campbell S, Michaelson J, Burkly L, Putterman C: The role of TWEAK/Fn14 in the pathogenesis of inflammation and systemic autoimmunity. Front Biosci 2004, 9:2273-2284

13. Chicheportiche Y, Chicheportiche R, Sizing I, Thompson J, Benjamin CB, Ambrose C, Dayer JM: Proinflammatory activity of TWEAK on human dermal fibroblasts and synoviocytes: blocking and enhancing effects of anti-TWEAK monoclonal antibodies. Arthritis Res 2002, 4:126-133

14. Kamata K, Kamijo S, Nakajima A, Koyanagi A, Kurosawa H, Yagita H, Okumura K: Involvement of TNF-like weak inducer of apoptosis in the pathogenesis of collagen-induced arthritis. J Immunol 2006, 177:6433-6439

15. Perper SJ, Browning B, Burkly LC, Weng S, Gao C, Giza K, Su L, Tarilonte L, Crowell T, Rajman L, Runkel L, Scott M, Atkins GJ, Findlay DM, Zheng TS, Hess H: TWEAK is a novel arthritogenic mediator. J Immunol 2006, 177:2610-2620

16. Kamijo S, Nakajima A, Kamata $K$, Kurosawa $H$, Yagita $H$, Okumura K: Involvement of TWEAK/Fn14 interaction in the synovial inflammation of RA. Rheumatology (Oxford) 2008, 47: 442-450

17. Park MC, Chung SJ, Park YB, Lee SK: Relationship of serum TWEAK level to cytokine level, disease activity, and response to antiTNF treatment in patients with rheumatoid arthritis. Scand J Rheumatol 2008, 37:173-178

18. van Kuijk AW, Wijbrandts CA, Vinkenoog M, Zheng TS, Reedquist KA, Tak PP: TWEAK and its receptor Fn14 in the synovium of patients with rheumatoid arthritis compared to psoriatic arthritis and its response to tumour necrosis factor blockade. Ann Rheum Dis 2010, 69:301-304

19. Dharmapatni AA, Smith MD, Crotti TN, Holding CA, Vincent C, Weedon HM, Zannettino AC, Zheng TS, Findlay DM, Atkins GJ, Haynes DR: TWEAK and Fn14 expression in the pathogenesis of joint inflammation and bone erosion in rheumatoid arthritis. Arthritis Res Ther 2011, 13:R51

20. Kong YY, Feige U, Sarosi I, Bolon B, Tafuri A, Morony S, Capparelli C, Li J, Elliott R, McCabe S, Wong T, Campagnuolo G, Moran E, Bogoch ER, Van G, Nguyen LT, Ohashi PS, Lacey DL, Fish E, Boyle WJ, Penninger JM: Activated T cells regulate bone loss and joint destruction in adjuvant arthritis through osteoprotegerin ligand. Nature 1999, 402:304-309

21. Ju JH, Cho ML, Moon YM, Oh HJ, Park JS, Jhun JY, Min SY, Cho YG, Park KS, Yoon CH, Min JK, Park SH, Sung YC, Kim HY: IL-23 induces receptor activator of NF-kappaB ligand expression on $\mathrm{CD} 4+\mathrm{T}$ cells and promotes osteoclastogenesis in an autoimmune arthritis model. J Immunol 2008, 181:1507-1518

22. Arnett FC, Edworthy SM, Bloch DA, McShane DJ, Fries JF, Cooper NS, Healey LA, Kaplan SR, Liang MH, Luthra HS, Medsger TA, Mitchell DM, Neustadt DH, Pinals RS, Schaller JG, Sharp JT, Wilder RL, Hunder GG: The American Rheumatism Association 1987 revised criteria for the classification of rheumatoid arthritis. Arthritis Rheum 1988, 31:315-324 
23. Cho ML, Kim WU, Min SY, Min DJ, Min JK, Lee SH, Park SH, Cho CS, Kim HY: Cyclosporine differentially regulates interleukin-10, interleukin-15, and tumor necrosis factor a production by rheumatoid synoviocytes. Arthritis Rheum 2002, 46:42-51

24. Kong YY, Yoshida H, Sarosi I, Tan HL, Timms E, Capparelli C, Morony S, Oliveira-dos-Santos AJ, Van G, Itie A, Khoo W, Wakeham A, Dunstan CR, Lacey DL, Mak TW, Boyle WJ, Penninger JM: OPGL is a key regulator of osteoclastogenesis, lymphocyte development and lymph-node organogenesis. Nature 1999, 397:315-323

25. Takayanagi $H$, Iizuka $H$, Juji $T$, Nakagawa $T$, Yamamoto $A$, Miyazaki T, Koshihara Y, Oda H, Nakamura K, Tanaka S: Involvement of receptor activator of nuclear factor kappaB ligand/ osteoclast differentiation factor in osteoclastogenesis from synoviocytes in rheumatoid arthritis. Arthritis Rheum 2000, 43:259-269

26. Kim KW, Cho ML, Lee SH, Oh HJ, Kang CM, Ju JH, Min SY, Cho YG, Park SH, Kim HY: Human rheumatoid synovial fibroblasts promote osteoclastogenic activity by activating RANKL via TLR-2 and TLR-4 activation. Immunol Lett 2007, 110:54-64

27. Hulsmans HM, Jacobs JW, van der Heijde DM, van AlbadaKuipers GA, Schenk Y, Bijlsma JW: The course of radiologic damage during the first six years of rheumatoid arthritis. Arthritis Rheum 2000, 43:1927-1940

28. Lindqvist E, Jonsson K, Saxne T, Eberhardt K: Course of radiographic damage over 10 years in a cohort with early rheumatoid arthritis. Ann Rheum Dis 2003, 62:611-616

29. Lindqvist E, Saxne T, Geborek P, Eberhardt K: Ten year outcome in a cohort of patients with early rheumatoid arthritis: health status, disease process, and damage. Ann Rheumatic Dis 2002, 61:1055-1059

30. Polek TC, Talpaz M, Darnay BG, Spivak-Kroizman T: TWEAK mediates signal transduction and differentiation of RAW264.7 cells in the absence of Fn14/TweakR: evidence for a second TWEAK receptor. J Biol Chem 2003, 278:32317-32323 